\title{
A CONVERSE OF BANACH'S CONTRACTION THEOREM
}

\author{
LUDVIK JANOS
}

1. Introduction. Let $(S, \rho)$ be a bounded complete metric space and $\phi$ a contractive mapping of $S$ into itself; i.e.,

$$
\rho(\phi(x), \phi(y)) \leqq \alpha \rho(x, y), \quad \text { where } \alpha \in(0,1), \quad x, y \in S .
$$

Then it follows from a theorem due to Banach that the iterated images, $\phi^{n}(S)$ of $S$ shrink to the fixed point, $a$ of $\phi$. This fact can be written in the form $\bigcap_{n=1}^{\infty} \phi^{n}(S)=\{a\}$.

Since this formula does not involve the metric and has a topological character, it is therefore natural to ask the following question.

Let $S$ be a compact metrizable topological space and $\phi$ a continuous mapping of $S$ into itself which has the property that $\cap \phi^{n}(S)=\{a\}$. Is it possible to find a metric $\rho(x, y)$ generating the given topology of $S$ such that the mapping $\phi$ is contractive with respect to $\rho$ ? The answer to this question is affirmative and we will give the construction of the desired metric $\rho$. It should be mentioned that in the paper [1] a similar problem has been solved for a given abstract set $S$ and a mapping $\phi: S \rightarrow S$ satisfying the condition that each iteration $\phi^{n}$ of $\phi$ has a unique fixed point.

2. Construction of the metric with respect to which $\phi$ is nonexpansive. We will assume $S$ to be a compact metrizable topological space, and denote by $\mathfrak{T}$ the set of all metrics on $S$ generating the given topology of $S$. The mapping $\phi$ will be assumed to be continuous on $S^{*}$ and to satisfy $\cap \phi^{n}(S)=\{a\}$.

2.1. TheOREM 1. Under the assumptions made on $\phi$, there exists in Ml a distance function $\bar{\rho}$ such that $\bar{\rho}(\phi(x), \phi(y)) \leqq \bar{\rho}(x, y)$.

Proof. Let us take any $\rho \in \mathscr{T}$ and define $\bar{\rho}(x, y)$ as follows. $\bar{\rho}(x, y)$ $=\sup _{n} \rho\left(\phi^{n}(x), \phi^{n}(y)\right)$, for $n=0,1,2, \ldots$. From the definition we have $\bar{\rho}(x, y) \geqq \rho(x, y)$. The triangle inequality follows easily. For let $x, y, z \in S$. From the definition of $\bar{\rho}$ there exists a number $n$ such that $\bar{\rho}(x, z)=\rho\left(\phi^{n}(x), \phi^{n}(z)\right)$, and

$$
\bar{\rho}(x, z) \leqq \rho\left(\phi^{n}(x), \phi^{n}(y)\right)+\rho\left(\phi^{n}(y), \phi^{n}(z)\right) \leqq \bar{\rho}(x, y)+\bar{\rho}(y, z) .
$$

In order to prove that $\bar{\rho} \in \mathfrak{N}$ we have only to show that $x_{n} \rightarrow \rho x$ implies $x_{n} \rightarrow \bar{\rho} x$. Let us assume that $\bar{\rho}\left(x_{n}, x\right) \rightarrow 0$. Then there exists a

Presented to the Society, November 29, 1963 under the title Converse of the theorem on contracting mapping; received by the editors December 17, 1964. 
subsequence of $\left\{x_{n}\right\}$, which we denote again by $\left\{x_{n}\right\}$, for which $\bar{\rho}\left(x_{n}, x\right) \rightarrow \gamma>0$, where $\gamma$ is some positive number. From the definition of $\bar{\rho}$ follows the existence of integers $k_{1}, k_{2}, \cdots$ such that $\bar{\rho}\left(x_{n}, x\right)$ $=\rho\left(\phi^{k_{n}}\left(x_{n}\right), \phi^{k_{n}}(x)\right)$ for $n=1,2, \cdots$. We have to consider two cases:

(a) The set $\left\{k_{n}\right\}$ is bounded.

Then there exists a number $k$ in the sequence $\left\{k_{n}\right\}$ which is infinitely repeated and therefore for a suitably selected subsequence we have $\rho\left(\phi^{k}\left(x_{n}\right), \phi^{k}(x)\right) \rightarrow \gamma>0$ which is a contradiction because $x_{n} \rightarrow x$ and therefore also $\phi^{k}\left(x_{n}\right) \rightarrow \phi^{k}(x)$.

(b) The set $\left\{k_{n}\right\}$ is not bounded.

Selecting a suitable subsequence we have the result that

$$
\rho\left(\phi^{k_{n}}\left(x_{n}\right), \phi^{k_{n}}(x)\right) \rightarrow \gamma>0
$$

where $\left\{k_{n}\right\}$ is now a monotonically increasing sequence of natural numbers. Because $\phi^{k_{n}}(x) \in \phi^{k_{n}}(S)$ and $\cap \phi^{k_{n}}(S)=\{a\}$ we arrive again at a contradiction, which proves our assertion.

3. The construction of a metric $\rho^{*}$ with respect to which the mapping $\phi$ is contractive.

3.1. Definition. Let $S=A_{0}, \phi(S)=A_{1}, \cdots, \phi^{n}(S)=A_{n} \cdots$ and introduce the functions $n(x)$ and $n(x, y)$ as follows:

$$
n(x)=\max \left\{n: x \in A_{n}\right\}, \quad n(x, y)=\min \{n(x), n(y)\} .
$$

3.2. Theorem 2. For any $\alpha \in(0,1)$ there exists in $\mathfrak{T}$ a distance function $\rho^{*}$ such that $\rho^{*}(\phi(x), \phi(y)) \leqq \alpha \rho^{*}(x, y)$.

Proof. By Theorem 1 there exists a metric $\rho(x, y)$, such that the mapping $\phi$ is nonexpansive with respect to it. Let

$$
\lambda(x, y)=\alpha^{n(x, y)} \rho(x, y) .
$$

Because $n(\phi(x), \phi(y))=n(x, y)+1$, it follows that

$$
\lambda(\phi(x), \phi(y)) \leqq \alpha \lambda(x, y) .
$$

The function $\lambda(x, y)$ is not in general a metric. However a desired metric $\rho^{*}(x, y)$ can be defined as

$$
\rho^{*}(x, y)=\inf \sum_{i=1}^{n} \lambda\left(x_{i}, x_{i+1}\right)
$$

where the infimum is taken over all possible finite systems of elements $x_{1}, x_{2}, \cdots, x_{n+1} \in S$ such that $x=x_{1}$ and $x_{n+1}=y$.

It follows from the definition that $\rho^{*}(x, y) \leqq \lambda(x, y) \leqq \rho(x, y)$. We will show the validity of the triangle inequality for $\rho^{*}$. Let $x, y, z \in S$ and $\epsilon>0$. From the definition of $\rho^{*}(x, y), \rho^{*}(y, z)$ there exist elements 
$u_{1}, u_{2}, \cdots, u_{n+1}, v_{1}, v_{2}, \cdots, v_{m+1}$ such that $u_{1}=x, u_{n+1}=y, v_{1}=y$, $v_{m+1}=z$ and

$$
\rho^{*}(x, y)=\sum_{i=1}^{n} \lambda\left(u_{i}, u_{i+1}\right)-\epsilon_{1}, \quad \rho^{*}(y, z)=\sum_{i=1}^{m} \lambda\left(v_{i}, v_{i+1}\right)-\epsilon_{2},
$$

where $\epsilon_{1}, \epsilon_{2}<\epsilon$. From the definition of $\rho^{*}(x, z)$ we have

$$
\rho^{*}(x, z) \leqq \sum_{i=1}^{n} \lambda\left(u_{i}, u_{i+1}\right)+\sum_{i=1}^{m} \lambda\left(v_{i}, v_{i+1}\right) .
$$

Therefore we have

$$
\rho^{*}(x, y)+\rho^{*}(y, z) \geqq \rho^{*}(x, z)-\epsilon_{1}-\epsilon_{2} .
$$

In order to prove that $\rho^{*}(x, y)$ is a metric it remains to show that $\rho^{*}(x, y) \neq 0$ for $x \neq y$. Let $n(x) \leqq n(y)$. From the definition of $\rho^{*}(x, y)$ it can directly be seen that if $n(x)=n(y)$ then $\rho^{*}(x, y) \geqq \alpha^{n(x)} \rho(x, y)$ while if $n(x)<n(y)$ then $\rho^{*}(x, y) \geqq d(x) \cdot \alpha^{n(x)}$ where $d(x)$ is a distance of the point $x$ from the compact set $A_{n(x)+1} . d(x)$ is therefore a positive number, hence $\rho^{*}$ is a distance function. In order to prove that $\rho^{*} \in \mathscr{T}$ it remains to show that $x_{n} \rightarrow \rho^{*} x$ implies $x_{n} \rightarrow \rho x$. If this is not the case, then because of compactness with respect to $\rho$ there exists a subsequence, which we denote again by $\left\{x_{n}\right\}$ such that $x_{n} \rightarrow \rho y$. $y \neq x$, and therefore also that $\rho^{*}\left(x_{n}, y\right) \rightarrow 0$, which is a contradiction.

It remains to prove that $\rho^{*}(\phi(x), \phi(y)) \leqq \alpha \rho^{*}(x, y)$. Let $\epsilon>0$ be a given number. From the definition of $\rho^{*}(x, y)$, there exists a representation of $\rho^{*}(x, y)$ in the form

$$
\rho^{*}(x, y)=\sum_{i=1}^{n} \lambda\left(x_{i}, x_{i+1}\right)-\epsilon_{1} \quad \text { where } \epsilon_{1}<\epsilon .
$$

Now we have

$$
\rho^{*}(\phi(x), \phi(y)) \leqq \sum_{i=1}^{n} \lambda\left(\phi\left(x_{i}\right), \phi\left(x_{i+1}\right)\right)=\alpha \sum_{i=1}^{n} \lambda\left(x_{i}, x_{i+1}\right),
$$

which gives

$$
\rho^{*}(\phi(x), \phi(y)) \leqq \alpha \rho^{*}(x, y)+\alpha \epsilon_{1} .
$$

Because $\epsilon$ was chosen arbitrarily, this proves our theorem.

\section{REFERENCE}

1. C. Bessanga, On the converse of the Banach fixed point principle, Colloq. Math. 7 (1959), 41-43.

George Washington University and UNIVERSITY OF FLORIDA 